\title{
eJRIEPS
}

Ejournal de la recherche sur l'intervention en éducation physique et sport

$18 \mid 2009$

Varia

\section{Modalités de direction d'étude et apprentissage de l'attaque en volley-ball : quels effets de genre?}

\author{
Ingrid Verscheure
}

\section{OpenEdition}

Journals

Édition électronique

URL : http://journals.openedition.org/ejrieps/5517

DOI : 10.4000/ejrieps.5517

ISSN : 2105-0821

Éditeur

ELLIADD

\section{Référence électronique}

Ingrid Verscheure, «Modalités de direction d'étude et apprentissage de l'attaque en volley-ball : quels effets de genre? ", eJRIEPS [En ligne], 18 | 2009, mis en ligne le 01 juillet 2009, consulté le 18 mars 2021. URL : http://journals.openedition.org/ejrieps/5517 ; DOI : https://doi.org/10.4000/ejrieps.5517

\section{(c) (1)}

La revue eJRIEPS est mise à disposition selon les termes de la Creative Commons Attribution 4.0 International License. 
Modalités de direction d'étude et apprentissage de l'attaque en volley-ball : quels effets de genre?

Ingrid Verscheure

UFR STAPS Franche-Comté, LASELDI, France

\section{Résumé}

Différents travaux montrent que les dimensions sexuées influencent la façon dont chaque élève, fille ou garçon, interagit avec les situations didactiques proposées par leur professeur. Nos recherches ont confirmé les effets de l'action didactique conjointe des enseignants ${ }^{1}$ et des élèves sur la dynamique différentielle de construction des "positions de genre" en éducation physique. Dans le cadre de cet article, nous nous interrogeons sur le rôle de la direction d'étude dans la co-construction du savoir attaquer en volley-ball. Nous tentons d'en décrire ses effets en termes d'acquisitions différentielles selon le genre des élèves. Nous avons observé et comparé deux systèmes didactiques différents du point de vue du type d'enseignement mis en œuvre par le professeur. Les résultats montrent que l'intentionnalité didactique du professeur joue un rôle important dans les processus différentiels à l'œuvre. Les dynamiques mises en évidence soulignent la nécessité d'un enseignement des sports collectifs s'attachant explicitement aux dimensions à la fois techniques et tactiques du jeu si l'on souhaite une plus grande équité dans les acquisitions des filles et des garçons.

\section{Introduction}

Cet article rend compte d'une partie d'un travail de thèse portant sur la dynamique différentielle et la co-construction de la différence des sexes en éducation physique et sportive (EPS) à partir d'une analyse comparative de deux systèmes didactiques contrastés selon le type d'enseignement qui y est développé. Nous avons repris l'idée avancée par Johsua et Lahire (1999) qui regrettent que la didactique ne s'intéresse pas suffisamment aux dimensions sociales dans l'apprentissage des élèves, en nous nous intéressant plus particulièrement à l'aspect sexué des profils identitaires des élèves en relation avec l'enseignement qu'ils reçoivent. Si de nombreux travaux en langue anglaise et française ont mis en évidence les inégalités scolaires de sexe dans l'enseignement en général, mais aussi en éducation physique, peu d'entre eux se sont attachés à étudier

${ }^{1}$ Dans ce document, l'emploi du masculin pour désigner des personnes n'a d'autres fins que celle d'alléger le texte. 
dans le détail comment ces inégalités se construisent au fil des situations d'enseignementapprentissage, c'est-à-dire en prenant en considération le contenu enseigné. Dans notre travail, nous avons tenté de déterminer comment les dispositions sociales des élèves et notamment leurs identités sexuées se transforment ou non en dispositions scolaires en examinant comment différents indicateurs participent aux processus d'apprentissage différentiel: représentations, genre mesuré par l'Inventaire des Rôles de Sexe de Bem (IRSB, tel que adapté par Fontayne, Sarrazin et Famose, 2000), position de genre activée (Verscheure \& Amade-Escot, 2007), inscription dans le contrat didactique différentiel (Schubauer-Leoni, 1996). Dans cet article, nous intéressons plus particulièrement aux effets différentiels selon le genre des modes d'enseignement de l'attaque en volley-ball, en faisant l'hypothèse que la valorisation de telle ou telle composante de l'attaque (la dimension tactique, la dimension technique, l'exécution d'un modèle gestuel du smash, etc..) pouvait avoir des répercussions sur la manière dont les élèves de genre différents dans l'apprentissage et l'étude des contenus qui leur étaient proposés.

Pour en rendre compte, nous présentons rapidement le contexte scientifique des recherches sur les inégalités sexuées en éducation physique. Ceci nous amène à justifier le recours à l'approche didactique et présenter la problématique de notre recherche. Dans une seconde section nous développons les aspects méthodologiques. Nous exposons ensuite les résultats de l'étude de cas menée dans deux classes de $1^{\text {ère }}$ et les discutons avant de conclure.

\section{Cadre théorique et problématique}

Dans cette partie, nous présenterons un état des lieux sur les inégalités de sexe dans le système scolaire et plus particulièrement en éducation physique, selon différentes thématiques. Ensuite, après avoir indiqué l'intérêt d'une approche didactique pour comprendre les inégalités filles et garçons en EPS, nous présenterons la problématique et les questions ayant orienté notre recherche.

1. 1. Inégalités fille-garçons à l'école et en EPS

Ce travail trouve son ancrage dans les travaux sur les différences sexuées en éducation physique qui mettent en évidence que, dans un enseignement mixte, il persiste des inégalités de traitement des filles et des garçons ayant des répercussions sur leurs acquisitions, sur leur évaluation, et sur les apprentissages réalisés. La question de l'égalité entre les filles et les garçons dans le système scolaire est une question vive. Les conclusions des différentes études portant sur ce thème montrent que l'école continue à véhiculer une certaine image du masculin et du féminin, ce qui a pour conséquence 
d'importantes inégalités de trajectoires scolaires. De nombreux travaux en sociologie et psychosociologie (Duru-Bellat, 1994 ; Mosconi \& Loudet-Verdier, 1997) se sont intéressés à l'étude de la construction sociale des identités et des comportements sexués au sein du système scolaire. La mixité scolaire prend en compte la différence des genres et prépare par-là même aux rapports entre les sexes. La plupart des auteurs spécialistes de ce sujet tant dans la recherche anglo-saxonne que francophone estime que l'éducation physique participe à cette construction et renforce les représentations sexuées des acteurs relativement à la pratique des activités physiques et sportives et artistiques (APSA). De la revue de cette littérature fournie concernant le thème de la différence entre filles et garçons en éducation physique il ressort quatre grands axes de recherche aux résultats significatifs :

1) Les aspects organisationnels de l'enseignement en mixité. En France, le pourcentage de classes mixtes en EPS a augmenté sensiblement depuis 1979 et le taux de classes ou groupes mixtes décline de la $6^{\text {ème }}$ à la terminale (Davisse et Louveau, 1991, Cogérino, Terret, Rogowski, 2006). Cependant, alors même que les filles et les garçons de la classe ont cours ensemble, la mixité des pratiques peut ne pas être réelle. Par principe, les enseignants sont favorables à la mixité mais en pratique, la question des modalités de travail dans le champ des APSA, la signification des prestations physiques, de leur niveau constituent pour les enseignants et pour les élèves les butoirs de la mixité.

2) L'enseignement de l'éducation physique en classes mixtes. II reste donc problématique en raison notamment de représentations et attentes (meanings) différenciées des enseignants et des élèves (Davisse \& Louveau, 1991; Harris \& Penney, 2000 ; Macdonald, 1990 ; Wright, 1997). Selon qu'ils s'adressent à des élèves filles ou des élèves garçons les enseignants, de façon inconsciente, développent des différences d'objectifs, de traitement et d'interactions. II existe aussi des différences entre les activités proposées par les enseignantes et celles proposées par les enseignants (Harris, 1997). De plus, les élèves abordent les APSA selon des perceptions et représentations sexuées (Luke \& Sinclair, 1991). II existe des différences physiques, morphologiques et physiologiques indéniables entre les filles et les garçons. Une fois intériorisées, ces différences sont plus ou moins institutionnalisées et renvoient à une hypothèse pérenne à savoir que les filles sont plus faibles que les garçons (Davisse, 1991). L'enseignement en éducation physique (politique et pratique) renforce de façon implicite la différence des genres, en termes d'activités proposées mais également par les attitudes et réactions qui en découlent (Scraton, 1986). Selon Wright (1997), les élèves et les professeurs 
reproduisent ensemble la construction de genre dans leur pratique quotidienne de l'éducation physique.

3) Le marquage sexuel de pratiques physiques. Cet axe a été initié par Davisse \& Louveau (1991). Les APSA sont perçues comme différemment appropriées aux filles ou aux garçons (Cleuziou, 2000, Fontayne, Sarrazin \& Famose, 2001). Pour pallier les problèmes complexes engendrés par la mixité, certains enseignants proposent des APS « appropriées aux deux sexes » (Fontayne et al., 2001), notamment les APPN (Davisse, 1991) voire des APS « nouvelles » (base-ball ou ultimate) afin de mettre les élèves sur un pied d'égalité par rapport au peu de vécu dans cette APS ainsi qu'à la méconnaissance des règles. Cependant, à l'entrée du lycée les filles sont largement impliquées dans les pratiques sportives et leurs attitudes face au sport ainsi que leurs motivations rejoignent celles des garçons. Ainsi, certaines auteures (Couchot-Schiex, 2007, Vigneron, 2004) montrent que ce serait au niveau des tâches d'apprentissage que se jouent les différenciations sexuées. Identifiant des difficultés techniques chez les filles, les enseignants ne proposent pas spontanément des contenus qui permettraient de les résoudre mais s'appuient sur des apprentissages sociaux, comme si cela suffisait.

4) Le choix des activités. Cet axe invite à prendre en considération les formes de traitement didactique et le contenu spécifique des leçons d'éducation physique, qui jouent un rôle déterminant pour développer une éducation physique moins sexiste (Davisse, 1991 ; Kirk, Brooker \& Braiuka, 2000 ; Marsenach, 2004 ; Vigneron, 2004). II ressort de ces travaux récents que les contenus proposés aux élèves ne sont pas neutres.

Les conclusions de ces quatre axes d'études rejoignent les réflexions mettant en avant la nécessité de développer des analyses très précises incluant le point de vue didactique en prenant en considération les rapports sexués aux savoirs scolaires comme variable explicative des différences d'implication et d'acquisition entre filles et garçons (AmadeEscot, 2005 ; Verscheure \& Amade-Escot, 2004, Mosconi, 2003). Rappelons aussi que l'enquête de Fize (2003) montre combien il est difficile de cadrer le sujet de la mixité scolaire en prenant en compte tous les paramètres afin de ne pas se satisfaire des représentations communes remettant petit à petit en cause le bien fondé de la mixité scolaire. Pour ce qui concerne les sports collectifs, nous rejoignons Bergé (2004) et défendons l'idée que les mises en œuvre des compétences stratégiques et tactiques sont différentes selon les filles et les garçons mais qu'elles doivent être également valorisées (nous y reviendrons). Ce point de vue nous semble souligner l'intérêt des analyses qui examinent la façon dont les élèves investissent les APS qui dépassent les clivages sexués 
pour inclure les niveaux de pratique et les habiletés momentanées disponibles par les uns et les autres (Marsenach, 2004).

Au terme de cette revue de littérature, nous pouvons considérer qu'il existe un consensus décrivant les inégalités de traitement et de réussite entre filles et garçons, en EPS et à l'école. Malgré quelques travaux encore pionniers que nous venons de citer, il reste que les explications avancées par les différents chercheurs laissent certains aspects des processus dans l'ombre. Peu d'informations précises sont disponibles quant à la façon dont, dans la vie quotidienne de la classe, les filles et les garçons s'approprient les contenus de l'EPS. II semble donc intéressant d'approfondir la question des contenus transmis à l'école en relation avec la manière avec laquelle ils sont véhiculés par les enseignants. Ceci impose d'effectuer une analyse très fine de l'activité du professeur et des élèves engagés dans des interactions d'enseignement et d'apprentissage. Les travaux précédemment cités montrent bien qu'on ne peut plus ni analyser les processus d'enseignement-apprentissage en classe mixte sans prendre en considération les savoirs enseignés, ni considérer les élèves comme un groupe constitué d'éléments "identiques ». Ceci nous a amené à re-problématiser la question des inégalités de sexe en EPS dans le champ de la didactique, c'est-à-dire aller voir dans le détail des interactions en situation ce qui s'y déroule, en prenant en compte le savoir et notamment la connotation masculine ou féminine des APSA.

1. 2. Re-problématisation de la question des inégalités de sexe en EPS dans le champ de la didactique

Rappelons que les recherches didactiques visent à décrire, comprendre et expliquer les activités scolaires qui relèvent de la transmission-appropriation des savoirs disciplinaires. Cette approche se centre sur les contenus d'enseignement et étudie les conditions et les contraintes qui sont à prendre en compte pour que les élèves se les approprient. Les savoirs réellement enseignés et appris dans cette perspective sont le résultat d'une action conjointe entre professeur et élèves (Amade-Escot, 2007 ; Marsenach et Mérand, 1987 ; Sensevy et Mercier, 2007). Ils émergent au fil des interactions professeurs et élèves en relation avec le contenu des situations d'apprentissage. Dans cette perspective théorique, l'activité du professeur consiste à diriger l'étude des élèves. A la suite de Chevallard (1997), nous définissons la "direction d'étude" par deux grands types de tâches : l'enseignant doit d'une part, agencer des milieux didactiques adaptés en proposant aux élèves des situations d'apprentissage, d'autre part, gérer au fil des séances l'interaction des élèves à ce milieu et ce, en relation avec son intentionnalité didactique, sa conception du savoir à enseigner, les buts de tâches valorisés, les consignes données, etc. Il convient 
de souligner que l'étude (des élèves) et la direction d'étude (par le professeur) résultent de processus adaptatifs conjoints, d'où le caractère émergeant des contenus d'enseignement et le caractère situé des processus didactiques. S'attacher à comprendre la direction d'étude en contexte d'enseignement de l'éducation physique et sportive pour identifier ses effets sur les apprentissages différentiels des élèves selon la position de genre qu'ils activent est le but de cet article. L'action en contexte étant indissociablement matérielle (artéfact), sociale et culturelle, nous avons considéré que la mise en place des tâches, le type de savoirs mis à l'étude, les techniques didactiques utilisées pour réguler l'activité des élèves jouent un rôle important et influent sur la direction d'étude du professeur et, par voie de conséquence, sur l'étude des élèves au sein de chaque système didactique observé.

Le système didactique de l'éducation physique est le « système de relation entre le jeu de l'enseignant et le jeu de l'élève à propos du savoir à enseigner et à apprendre, sous couvert d'institutions dotées d'intention d'enseignement » (Schubauer-Leoni, 1998, p.334). Il est conçu comme un système dynamique de transformation de savoirs, des pratiques et des connaissances au sein d'institutions ayant pour mission le projet social de leur transmission. Dans ce cadre, il a été établi que le travail didactique de l'enseignant vise à maintenir la relation des élèves avec le savoir enseigné (Amade-Escot, 1998) alors même que les contenus de l'EPS sont des objets instables. C'est pourquoi les compétences didactiques des enseignants se développent au sein d'un système d'interactions contextualisées fortement déterminées par les particularités des contenus enseignés. Cette auteure a montré que l'activité didactique de l'enseignant d'EPS est adaptative et contextualisée aux savoirs qui sont « en jeu » et « l'enjeu » des situations.

Dans cette perspective, le concept de contrat didactique apparaît central. C'est un « toujours-déjà-là » qui institue la relation didactique par la réunion des trois pôles, distribuant à chacun son rôle. Bien qu'implicite, ce contrat suppose le principe d'un consentement mutuel, quoique non formellement délibéré des contractants. Dans ce cadre de l'observation en contexte de situation d'enseignement-apprentissage, le contrat didactique définit "Ces habitudes (spécifiques) du maître attendues par l'élève et les comportements de l'élève attendus par le maître, c'est le contrat didactique" (Brousseau, 1980, p.180). Pour cet auteur l'évolution du contrat didactique est liée aux interactions qui se déroulent au cours de la séquence entre le professeur et les élèves (ou groupe d'élèves) autour d'un savoir précis. En EPS, le fonctionnement du contrat didactique a fait l'objet de plusieurs recherches (Amade-Escot, 2005 ; Goirand, 1998 ; Marsenach, 1995) qui ont mis en évidence que, lors de la phase interactive, à l'occasion des communications 
didactiques (celles qui donnent des informations sur ce qu'il y a à faire et sur la façon de le faire), de nombreux aléas modifient le contenu enseigné à l'insu du professeur comme des élèves. II ressort de ces études qu'observer l'évolution du contrat didactique in situ permet de rendre compte du processus d'enseignement-apprentissage, dans la mesure où il est lié au savoir enseigné. Ce contrat très local, car négocié lors d'une situation particulière entre l'enseignant, un élève (ou groupe d'élèves) et un contenu d'enseignement permet aussi de situer chaque élève au regard des apprentissages visés. En effet, et comme l'a mis en évidence Schubauer-Leoni (1996), le contrat didactique n'est pas négocié uniquement entre l'instance du maître et celle que représente l'ensemble des élèves, mais entre le maître et des sous-groupes d'élèves correspondant à diverses positions scolaires au sein de la classe. Nous avons fait l'hypothèse que des phénomènes similaires peuvent exister en relation avec la différence des sexes. En suivant le postulat d'un contrat didactique différentiel, nous considérons que les sujets enseignés, selon qu'ils occupent une place d'élèves ( forts », "faibles » voire " moyens », ou « filles » et " garçons », d'orientation de genre différente) ne se positionneront pas - et ne seront pas sollicités - de la même façon par le professeur qui interpellera l'un ou l'autre selon la fonction que chacun aura à remplir à tel moment d'avancement de l'activité. Schubauer-Leoni (2002) souligne par ailleurs que le contrat didactique est un contrat différentiel déterminant non seulement la représentation que le maître se construit des élèves appartenant aux différentes catégories sociales, mais aussi celle des élèves en situation d'apprentissage en fonction des attentes qu'ils identifient, affectant ainsi le savoir réellement enseigné. Ces différents éléments théoriques nous ont amené à retenir le concept de contrat didactique dans son acception différentielle pour identifier et comprendre les phénomènes de coconstruction des savoirs en classe dans leurs aspects différentiels selon les sexes (Verscheure \& Amade-Escot, 2007).

\section{3. Problématique et question de recherche}

A la suite des ces travaux nous avons considéré que les représentations des APS jouent un rôle déterminant dans la façon dont les élèves (filles et garçons, en fonction de leur genre) décodent les significations des tâches qui leur sont proposées. Une enquête préalable menée dans l'ensemble des Lycées d'Enseignement Généraux et Technologique Agricoles (LEGTA) de l'Académie de Toulouse a permis de caractériser les représentations des filles et des garçons à propos de l'éducation physique, des sports collectifs et du volley-ball (Verscheure, Amade-Escot \& Chiocca, 2006). Dans un second temps, nous avons observé le fonctionnement du système didactique dans deux des classes enquêtées afin d'observer les dynamiques différentielles de (co-)construction des 
savoirs en situation d'enseignement-apprentissage de l'attaque en volley-ball. Dans le cadre de cet article, nous rendons compte plus particulièrement d'un aspect de cette étude de terrain concernant l'influence des modalités de direction d'étude des deux enseignants observés sur les dynamiques d'apprentissage des élèves.

Nous avons retenu le volley-ball en tant qu'APS dite "appropriée aux deux sexes » (Fonatyne, Sarrazin \& Famose, 2001) souvent enseignée et appréciée à la fois par les enseignants et les élèves. Pour permettre la comparaison nous avons observé plus particulièrement deux tâches portant sur l'attaque en volley-ball et visant "la rupture de l'échange". Le protocole méthodologique qui sera décrit dans la section suivante prend appui sur les acquis de la littérature des "gender studies" en éducation physique pour prendre en compte les différences entre les élèves de genre différents non pour les figer mais pour créer les conditions de leur évolution, tout en préservant une exigence identique concernant les compétences stratégiques et tactiques (Bergé, 2004). La question de recherche que nous approfondissons ici est de nous interroger sur le rôle de la direction d'étude (au sens de Chevallard, 1997) dans la co-construction du savoir attaquer en volley-ball. Nous tentons d'en décrire - au fil des interactions didactiques - ses effets en termes d'acquisitions différentielles selon le genre des élèves.

\section{Méthode}

Nous indiquons les grandes lignes méthodologiques de recueil et de traitement des données mis en œuvre. Deux études empiriques emboîtées ont été mises en œuvre. Comme nous l'évoquions précédemment la première avait pour fonction de repérer les liens éventuels entre le genre des élèves mesuré par l'IRSB et les représentations de l'attaque en volley-ball (Verscheure, \& al., 2006). La seconde, dont nous rendons compte, ici est de type qualitatif et met en œuvre des procédures permettant de retrouver le sens des phénomènes en intégrant le temps, l'espace et les contextes locaux. Nous nous situons dans la perspective méthodologique maintenant devenue classique d'observation du didactique décrite par Leutenegger (2003) comme relevant d'une « clinique/expérimentale ». II s'agit d'analyser dans le détail l'évolution des interactions entre le professeur et certains élèves retenus ici en raison de leur identité de genre mesuré par l'IRSB (composante clinique) relativement à deux tâches d'apprentissage proposées par la chercheure (composante expérimentale que nous décrivons dans les sections suivantes).

Nous avons observé et comparé deux systèmes didactiques contrastés du point de vue du type d'enseignement de l'attaque en volley-ball mis en œuvre par leur enseignant à partir 
des deux tâches que nous leur avions demandé d'introduire dans leur cycle d'enseignement. Dans chacun des sites, nous avons retenus 8 élèves désignés comme représentatifs de la diversité de leur classe par les enseignants dont le score à l'IRSB avait été recueilli et pour lesquels nous disposions de données relatives à leur représentation de l'attaque en volley-ball (a partir des résultats de la première étude)

Au total, nous nous focalisons sur 2 enseignants et 16 élèves, observés in situ (au sein de leur collectif en fonctionnement) et aux prises avec des tâches ciblées. Ces acteurs sont considérés comme des révélateurs privilégiés de l'écologie de la séance en ce qu'elle produit des types de rapports à des contrats didactiques distincts. Ce sont ces éléments de contraste qui intéressent notre étude. Pour avancer les liens entre écologie de la séance et contrat didactique, nous nous appuyons sur le chapitre relatif au paradigme de l'écologie de la classe dans lequel Hastie et Siedentop (2006) font référence aux recherches françaises sur le contrat didactique et ses ruptures (p. 220) pour souligner les continuelles négociations à l'œuvre dans les classes d'éducation physique. Par ailleurs, les compatibilités théoriques entre ces deux courants de recherche ont été aussi récemment soulignées (Amade-Escot \& Venturini, 2009).

Avec Wirthner et Schneuwly (2004), nous pensons que le fait d'introduire un outil (ici les tâches d'attaque de la composante expérimentale) va transformer l'activité de l'enseignant qui l'utilise. Ceci paraît être un moyen pour contrôler et transformer ses propres systèmes de conception. Selon ces auteurs, lorsque le chercheur présente une situation « expérimentale » à un enseignant, il lui donne en fait une partition qu'il devra interpréter en construisant sa propre signification de la tâche et des enjeux d'apprentissage qui y sont associés. Dans l'approche "clinique/expérimentale", le chercheur examine comment l'enseignant interprète la composante expérimentale dans ses interactions avec les élèves. La mise en évidence de certaines façons de faire récurrentes chez les enseignants permet de saisir la signification globale de son action. Wirthner \& Schneuwly appellent « leitmotiv, le fil conducteur dominant de la séquence, la dimension récurrente essentielle à laquelle l'enseignant recourt pour donner cohérence à sa séquence » (2004, p.116). Dans notre étude, l'analyse didactique des modifications introduites permettra de comprendre au fil des observations la posture globale de l'enseignant dans l'appropriation de cette partition et repérer jusqu'où il entre dans la logique qui lui est proposée. Elle rend possible par ailleurs le repérage des savoirs « réellement » enseignés et des objets d'enseignement réellement mis à l'étude par l'enseignant notamment, comme nous le verrons ci-après les dimensions sexuées du savoir qu'il met à l'étude et la façon dont il dirige l'étude des 8 élèves observés. 
2. 1. Terrain de l'étude

Pour des raisons de faisabilité, l'observation s'est déroulée dans deux classes de $1^{\text {ère }}$ de deux LEGTA publics toulousains, réputés sportifs, notamment en UNSS rugby. Les deux enseignants que nous appellerons P-ALB et P-AUZ, ont accepté de nous recevoir dans leur classe pour être filmés et de participer aux différents entretiens. Nous avons choisi la période charnière de la classe de $1^{\text {ère }}$ où les enjeux d'apprentissages sont au centre du vécu des élèves et où il existe une certaine homogénéité des habiletés motrices.

2. 2. Choix de l'objet d'étude : attaque en volley-ball

La comparaison des APSA du point de vue du typage sexuel indique que volley-ball est le seul sport collectif «approprié aux deux sexes» (Fontayne et al. 2001), pratiqué couramment en LEGTA. Selon la littérature, les élèves filles semblent plus à l'aise dans cette APSA. Elles ont moins cette peur du contact, les déplacements semblent moins coûteux et le ballon peut sembler indolore. Davisse et Louveau (1991) précisent que la relation pédagogique y paraît plus aisée : le rapport entre la violence et la règle ainsi que les écarts de motivation entre filles et garçons peuvent y rester plus masqués. C'est pourquoi cette activité collective est enseignée la plupart du temps de façon mixte. Par ailleurs, le volley-ball s'inscrit tout à fait dans les finalités et objectifs de l'EPS au lycée, et l'attaque du camp adverse est une notion centrale du programme officiel de la classe de $1^{\text {ère }}$ (BO Hors-Série $n^{\circ} 6$ du 31 août 2000).

La logique du volley-ball consiste en un rapport d'opposition. Le but du jeu est de faire tomber le ballon dans le camp adverse et de l'empêcher de toucher le sol de son propre camp en l'envoyant réglementairement par-dessus le filet. Pour gagner un échange, il faut soit faire tomber le ballon dans le terrain adverse, soit obtenir que l'adversaire commette une faute. Les élèves doivent apprendre à jouer le ballon « avec » des partenaires et « contre » des adversaires. En ce qui concerne l'attaque, le rappel de ce point de règlement permet de dire qu'elle ne se limite pas au geste conclusif (le smash ou le renvoi) mais englobe le fait d'avoir une intentionnalité consistant à marquer le point à partir d'une construction collective. Cette phase de jeu est facilement observable et les différents comportements des élèves en attaque peuvent être décrits précisément. Enfin, il existe plusieurs modalités de conclusion de l'attaque (attaque smashée et attaque placée à deux mains) qui toutes deux peuvent permettre de rompre l'échange. En fonction des situations de jeu, les élèves s'inscrivent dans une intention collective et/ou individuelle d'attaque qui peut donc être observée dans ses différentes variations.

La littérature sur les inégalités de sexe en éducation physique ainsi que les travaux concernant les représentations des élèves sur l'attaque en volley-ball montrent que les 
garçons sont plutôt dans une visée de rupture de l'échange en force (smash) alors que les filles se situent plutôt dans une optique de continuité de l'échange (Davisse, 1991; Tanguy, 1992). Ce dernier montre que le mode d'entrée dans l'activité est différent selon les filles et les garçons. La plupart des filles s'efforcent simplement de se passer la balle par-dessus le filet, jouant « avec » les adversaires et non pas « contre ». L'important est de ne pas laisser tomber la balle; c'est le plaisir dans la durée, dans la continuité de l'échange. Pour les garçons, l'important c'est de faire le point, c'est le plaisir à court terme, c'est arrêter l'échange, la rupture de l'échange. Ceci est confirmé par plusieurs témoignages d'enseignants qui jugent que les représentations du volley-ball sont différentes selon le sexe des élèves. Les filles trouveraient que le ballon fait mal, attendant qu'il vienne à elle tandis que les garçons chercheraient à frapper le plus fort possible (Revue Contre Pied, 2005). Ainsi, bien que le volley-ball soit un sport collectif « approprié aux deux sexes », de subtiles différences selon le type d'entraînement vécu amènent des variations sur les représentations des joueurs filles et garçons. Bergé (2004) souligne que les mises en œuvre sont différentes selon les filles et les garçons mais qu'elles doivent être étiquetées comme étant de même valeur par l'enseignant. Créer la rupture en jouant prioritairement placé ou en jouant prioritairement en puissance (smash) sont deux mises en œuvre différentes d'une même compétence motrice (rompre intentionnellement l'échange au profit de son équipe). L'une n'a pas à être valorisée plus que l'autre ; les deux sont à construire en situation d'apprentissage scolaire. Ainsi, l'enseignement devrait permettre à la majorité des filles de s'appuyer sur des secteurs de jeu où elles sont compétentes (jouer là où l'adversaire n'est pas) pour acquérir le jeu fort et rapide (frappe puissante pour mettre l'adversaire en crise de temps pour renvoyer). Le chemin inverse est à construire pour les élèves, majoritairement des garçons, qui privilégient le jeu rapide. Le « jeu placé » ne doit pas être considéré comme inférieur au " jeu rapide » même s'il apparaît moins spectaculaire aux yeux de l'enseignant et des élèves.

C'est pourquoi, par l'intermédiaire de tâches proposant de travailler l'attaque du camp adverse, dans sa dimension de puissance (smash) et/ou de précision (frappe placée à 1 main ou en passe à 2 mains), nous chercherons à repérer comment et quels sont les élèves qui s'engagent dans l'un ou l'autre des chemins, quelles explications ou justifications ils en donnent, avec quelles conséquences implicites sur la dynamique différentielle du contrat didactique.

2. 3. Déroulement de l'étude en classe

Le protocole expérimental retenu a consisté à demander aux enseignants d'introduire dans un cycle ordinaire de volley-ball deux tâches sur lesquelles nous disposions 
d'analyses didactiques nous permettant de bien préciser les savoirs en jeu. Ces tâches appartiennent au répertoire classique des exercices d'apprentissage du volley-ball. Leur caractéristique est de mettre en scène deux modalités de réponses disponibles, en termes d'alternative d'attaque. Nous avons demandé aux enseignants de mettre en œuvre au moins une fois chacune de ces tâches pendant leur cycle de volley-ball, au moment où ils le jugeaient opportun et de faire évoluer celles-ci comme bon leur semblait. Aucun ordre de présentation des tâches ne leur a été suggéré. Seules les contraintes matérielles (hauteur du filet de $2 \mathrm{~m} 24$ et taille des terrains : 4x6m.) étaient précisées. Finalement, dans les deux sites et selon les variations que nous étudierons (les "leitmotiv" au sens de Wirthner \& Schneuwly, 2004), les enseignants ont utilisé ces deux tâches pendant trois séances que nous avons observées.

2. 4. Caractéristiques de la composante expérimentale : les tâches proposées aux enseignants

Afin de comparer des élèves ayant des caractéristiques différentes dans un enseignement commun (l'attaque en volley-ball) nous avons proposé aux deux enseignants de mettre en place deux tâches d'attaque, dites « tâches expérimentales ». Ces deux tâches permettent de mettre à l'étude le même contenu d'enseignement : la rupture de l'échange en frappant le ballon (smash) ou en le plaçant à 2 mains. Le but commun est de marquer le point selon l'alternative d'attaque. Ainsi, suivant le contexte d'opposition et le rapport de force, chacune des tâches permet aux élèves de conclure l'attaque et de marquer le point en adéquation avec l'état du rapport de force entre les deux équipes. Mais l'intérêt de ces deux modalités d'attaque est aussi de rendre compte des positions de genre susceptibles d'être activées par les élèves ou valorisées par les enseignants. En effet, chacune des deux formes possibles de l'alternative d'attaque - si elle est systématiquement mise en œuvre ou valorisée indépendamment du rapport de force - peut être analysée comme ce qui est habituellement décrit comme un stéréotype sexué en sport collectif (Davisse \& Louveau, 1991 ; Tanguy, 1992). En effet, la plupart des auteurs de la littérature anglosaxonne et francophone qui se sont intéressés aux stéréotypes sexués en sport ont mis en évidence les liens entre la valorisation des actions en puissance et en force et les stéréotypes masculins. Ils ont également montré que l'idée selon laquelle précision, jeu en continuité, ou encore la composante esthétique seraient liées à la pratique féminine témoigne d'un véritable stéréotype sexiste (Davisse, 1999 ; Davisse \& Louveau, 1991 ; Griffin, 1984, 1985 ; Kirk, 2002 ; Whitson, 1994). La composante « placer le ballon à 2 mains dans l'espace libre » peut véhiculer des modalités d'actions proches du jeu de renvoi qui serait l'apanage des filles alors que la composante « rompre l'échange en 
frappant le ballon » (smasher) peut correspondre au stéréotype masculin de l'attaque en force.

Pour conclure, dans ces deux tâches, même si les mises en œuvre cognitives et physiques sont différentes, la finalité reste la même : la construction tactique de la rupture de l'échange sans valorisation d'un ou de l'autre des alternatives. Elles permettent en principe de faire participer de manière égale les élèves ayant des représentations différentes, sans dénaturer la logique interne du volley-ball et le principe d'attaque du camp adverse trop souvent euphémisé lorsqu'il s'agit d'aborder ce thème, notamment dans les groupes de filles (Davisse, 1991). Par ailleurs, elles offrent aussi la possibilité d'observer les manières dont les deux enseignants et les différents élèves observés les interprètent, en permettant le repérage de positions de genre activées par chacun des acteurs de la relation didactique. Enfin, ces deux tâches concernent deux phases différentes de l'attaque. L'une est davantage centrée sur la phase de construction d'attaque, l'autre sur la conclusion de l'attaque en fonction du placement des adversaires, comme nous le précisons dans la section suivante.

2. 4. 1. La tâche «marquer le point en visant une des 2 zones laissées libres par l'adversaire après une réception sur lancer facile »

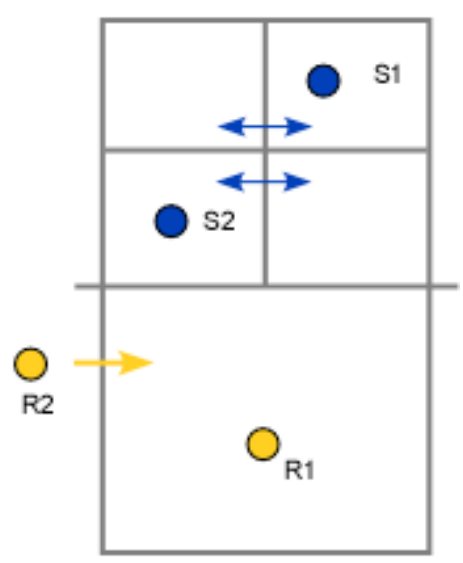

\section{Consignes}

- Mise en jeu de S1 sur R1.

- R2 rentre après que $\mathrm{R} 1$ ait touché le ballon.

Après la mise en jeu, l'équipe du serveur se place dans 2 des 4 zones et $y$ reste

- L'équipe des réceptionneurs doit viser une des zones restées libres (3 points)

Figure 1. Tâche expérimentale «marquer le point en visant une des 2 zones laissées libres par l'adversaire après une réception sur lancer facile » : tâche " viser »

Pour alléger le texte, cette tâche sera désignée sous le nom de 'tâche "viser "'. Elle consistait à attaquer le ballon en le plaçant dans une des deux zones laissées libres après une réception sur lancer facile.

L'analyse didactique de cette tâche (ou analyse a priori, Mercier \& Salin, 1988) révèle que le savoir en jeu dans cette tâche porte sur la liaison construction d'attaque dans son camp et l'efficacité du geste conclusif dans sa dimension lecture du placement adverse. Ici, 
l'équipe en réception/attaque doit s'organiser collectivement pour amener l'attaquant en position favorable. La réalisation de ce but dépend de l'état du rapport de force entre le lanceur et l'équipe en réception. Si R1 reçoit un ballon facile et est capable de faire une passe relativement précise sur son partenaire, la situation est facilitée. De son côté, R2 peut s'apercevoir très tôt de la difficulté de la balle et enclencher un déplacement visant à soutenir R1 (Amade-Escot, 2001). L'enjeu des transformations est la coordination des actions de deux joueurs de façon à placer l'attaquant en position favorable (balle haute, près du filet) lui donnant le temps de prendre des informations sur le placement des adversaires afin de placer la balle dans les deux espaces laissés libres. La variable de commande est liée au placement de S1 et S2 dans 2 des 4 zones, dégageant ainsi des espaces libres permettant la rétroaction du milieu. Si les élèves n'appliquent pas la consigne et prennent la balle qui arrive dans une des zones laissées initialement libre, alors le critère de réussite n'opère plus. Précisons que les serveurs, partenaires du milieu, choisissaient leurs zones de placement au moment de la réception de $\mathrm{R} 1$, avec pour seule obligation de rester dans la zone choisie.

2. 4. 2. La tâche « marquer le point face à deux adversaires (sur simple lancer de son passeur) selon l'alternative d'attaque »

Pour ne pas surcharger le texte, cette tâche sera désignée sous le nom de 'tâche " marquer "'. Dans cette tâche, les élèves devaient marquer le point face à deux défenseurs après 1 lancer.

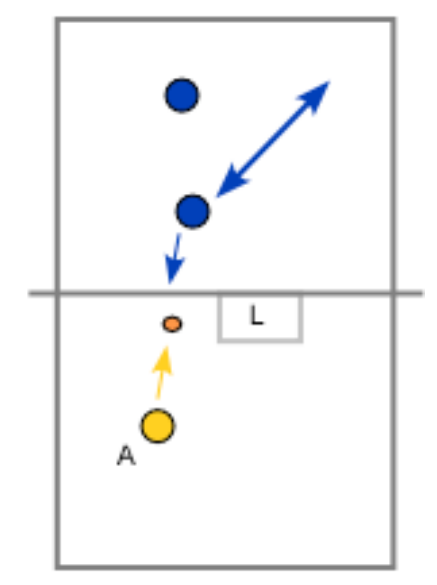

Figure 2. Tâche expérimentale «marquer le point face à deux adversaires selon

\section{Consignes}

- Attaque: L lance un ballon d'attaque, que l'attaquant $A$ doit passer de l'autre coté du filet en cherchant à marquer un point.

- Défense : 2 défenseurs, 1 déjà placé dans la zone arrière l'autre au filet. Ce dernier a le choix entre reculer pour faire une défense basse ou contrer (défense haute). La consigne donnée par le professeur est de varier les choix (contrer et reculer).

\begin{abstract}
Figure 2. Tâche expérimentale « marquer
l'alternative d'attaque » : tâche « marquer »
\end{abstract}


L'analyse a priori dévoile que le savoir en jeu dans cette tâche très classique porte sur l'efficacité de l'acte conclusif d'attaque. L'élève-attaquant doit prendre des informations sur les défenseurs pour décider où il doit mettre le ballon (loin d'eux). II doit s'organiser au plan moteur pour être efficace soit en plaçant la balle à 2 mains soit en smashant. II doit également juger (en fonction du ballon envoyé par le lanceur) si la balle est jouable en smash ou si elle est trop basse ou trop loin du filet, il est préférable de la placer à 2 mains. La tâche permet de construire le savoir-faire technique " placer ou frapper » en fonction du placement adverse (variable de commande). La rétroaction du milieu est immédiate, la variable de score (3 points si aucun des défenseurs ne touche le ballon ; 1 point si un des défenseurs touche le ballon mais ne peut pas la renvoyer) permet de valoriser l'intention tactique visant à attaquer là où ne sont pas les adversaires.

Rappelons que la visée de ces deux tâches était de mettre à l'étude les deux formes d'attaque efficace : soit l'attaque placée (susceptible de prendre en compte la dimension féminine d'engagement dans l'attaque), soit l'attaque en puissance (qui permettait de jouer sur la dimension masculine). D'une manière différente, ces deux tâches avaient pour but de faire travailler la notion de prise d'information sur l'adversaire et sur le ballon envoyé et valorisaient l'adaptation au rapport de force.

\section{5. Recueil des données}

Conformément aux procédures en usage dans la perspective méthodologique dite de « clinique/expérimentale » (Leutenegger, 2003), le recueil des données comprend différentes étapes. Nous avons tout d'abord mené un entretien de début de cycle avec chaque professeur participant à l'étude pour recueillir des informations sur ses conceptions à propos de l'enseignement du volley-ball. Ensuite, lors d'un entretien anteséance, nous avons investigué quelles étaient ses intentions didactiques pour chaque séance, que nous avons enregistré de façon audio et vidéo. A l'issue de chaque séance, un entretien avec le professeur a permis de recueillir son point de vue sur ce qui s'était passé et son avis sur les élèves observés. Du coté des élèves, à partir des données vidéos nous avons pu analyser leurs comportements en attaque dans les tâches de composante expérimentale. En cours de cycles, nous avons recueillis, leurs différentes interventions verbales auprès de leur camarade et en direction de leur professeur. Enfin, à la fin du cycle, nous avons effectué un entretien avec les élèves et avec le professeur. Ces entretiens avec chacun des acteurs permettent de rendre compte de leurs rapports aux objets et aux autres partenaires de la relation didactique. Ce recueil de données permet de constituer, sur chaque site, différents corpus (entretiens avec l'enseignant, entretiens avec les élèves, données de l'observation). 
2. 6. Trainement des données

L'analyse qualitative résulte d'un croisement entre les observations didactiques et les éléments du discours des différents acteurs, afin de réunir des informations aussi nombreuses et aussi détaillées que possible en vue de saisir la totalité d'une situation (Lessard-Hebert, Goyette \& Boutin, 1996).

Sur chaque site et pour les trois séances au cours desquelles les tâches expérimentales ont été mises à l'étude par les enseignants, les comportements en attaque des élèves ont été systématiquement codés à partir d'une grille d'observation. La grille a permis de caractériser le type d'actions produites en relation avec le rapport de force et son résultat, dans les différents rôles liés à la construction d'attaque (réceptionneur, passeur, attaquant, selon les tâches). Ces éléments couplés aux interactions verbales avec le professeur et les autres élèves ont permis d'établir une "chronique" pour chaque élève permettant de saisir l'évolution de leurs comportements en attaque, leur implication en lien avec la direction d'étude de leur enseignant (Verscheure \& Amade-Escot, 2007). Cette modalité de condensation des données par le biais de matrices qualitatives (les chroniques d'élèves) nous a permis de rendre plus lisibles et maniables les données recueillies, sans en perdre l'originalité ni la signification première (Huberman \& Miles, 1991).

Nous avons ensuite reconstruit (à partir de la triangulation des données d'observation et d'entretiens) le système des attentes réciproques entre l'enseignant et les élèves à propos du savoir enseigné au fil des trois séances. Cette deuxième étape du traitement des données rend compte de la dynamique différentielle du contrat didactique dans chaque site. Celle-ci évolue en relation d'une part avec le type de direction d'étude mis en œuvre par les deux enseignants, notamment la façon dont ils valorisent ou non certaines alternatives d'attaque (ou composantes) des tâches expérimentales. Elle évolue d'autre part en lien avec l'activation par les élèves d'une position de genre en adéquation (ou non) avec leur score à I'IRSB et leur représentation de l'attaque en volley-ball. Dans la suite de cet article, nous nous attachons à caractériser le type de direction d'étude des deux enseignants et à illustrer leurs effets à partir de quelques cas d'élèves de positions de genre contrastées.

\section{Résultats}

Nous avons synthétisé les données recueillies dans les deux systèmes didactiques (ALB et $A U Z$ ) pour examiner en quoi les conditions mises en place par les deux enseignants ont des répercussions sur les dynamiques différentielles d'apprentissage des élèves intégrant la question du genre. Nous nous sommes particulièrement intéressées à la variation de 
" direction d'étude », c'est-à-dire les dispositifs initiaux, les types de régulation, les composantes de tâches valorisées et leurs effets sur les conduites d'attaques de 16 élèves (6 filles et 10 garçons) telles qu'actualisées en situation.

Pour définir les systèmes didactiques observés, nous précisons les caractéristiques des deux enseignants, puis la trame du cycle prévue avant de développer les contenus concernant l'attaque en volley-ball des séances observées.

\section{1. Caractéristiques des deux enseignants}

P-ALB intervient dans un LEGTA récent qui dispose de nombreuses installations sportives. II est agrégé d'EPS et possède une dizaine d'années d'expérience. II se définit comme spécialiste du rugby et des sports collectifs. En UNSS, cet enseignant s'occupe des matchs de volley-ball mais également de ceux de rugby, ce qui lui permet d'instaurer un climat de confiance avec les élèves, propice au travail. Intéressé par notre projet, il a cependant émis des réserves en considérant que «tout le monde ne réussira pas à attaquer » (entretien ante-cycle).

Dans l'autre LEGTA, P-AUZ possède de nombreuses années d'expérience en tant qu'enseignant d'EPS. II cherche à instaurer un climat de confiance qui lui permet de mettre les élèves au travail. II discute beaucoup avec les élèves et s'intéresse à leurs activités scolaires et extrascolaires. Sa spécialité est le rugby mais il estime être également spécialiste des sports collectifs. Bien qu'intéressé par notre projet, il ne pensait pas que tous les élèves allaient réussir à « attaquer » (entretien ante-cycle).

\section{2. Trame des cycles prévus par les enseignants et séances observées}

Les entretiens ante-cycle et ante-séance nous ont permis de connaître ce que les enseignants avaient prévu de faire tout au long du cycle, et notamment le moment où s'intégrerait le travail de l'attaque.

\section{2. 1. Trame du cycle et séances observées à ALB}

Lors des 4 premières séances, le thème était le travail de la réception et la construction du jeu à 3 touches, en étant vigilant sur les notions de disponibilité et de mobilité pour ce faire. P-ALB cherche à rendre les élèves plus mobiles car ils ne sont concernés que lorsque le ballon arrive sur eux, en proposant des « situations d'opposition basées sur la mobilité et la disponibilité » (entretien ante).

L'enseignant commence le travail de l'attaque à la séance 5 . Selon lui, le thème de cette séance [obs. 1] est " jouer dans les espaces libres ». L'échauffement se termine par un match 1 contre 1 avec la consigne « regarder la position de l'adversaire pour jouer dans l'espace où il ne se trouve pas ». Puis il met en place la tâche expérimentale « Viser » qu'il va complexifier en ajoutant un défenseur, puis un attaquant. Les élèves doivent ensuite 
changer de rôle lorsque le ballon passe par-dessus le filet. Cette séance se clôt par une situation de jeu 3 contre 3 avec valorisation de l'attaque efficace « si le ballon tombe dans le camp adverse sans que l'adversaire ne le touche: 3 points». Cette connexité des tâches allant dans le sens d'une complication semble suivre une logique propre à ce professeur qui veut arriver la séance suivante à "prendre l'adversaire de vitesse ", « travailler le smash ». La séance 6 [obs. 2] s'organise autour du travail de la technique de manipulation de balle et notamment le geste du smash. Ce dernier est mis à l'étude dès l'échauffement avant d'être travaillé un long moment en situation « maximale " (facile, car le lanceur est fixe). L'enseignant fait réfléchir les élèves sur ce qu'il y a à faire pour réussir du point de vue de la coordination course d'élan/impulsion/frappe, liée à l'appréciation de la trajectoire de la balle et/ou l'appréciation du placement du défenseur. La séance se termine par une situation de jeu 4 contre 4 avec valorisation des points marqués en smashant. Lors de la séance 7 [obs. 3], l'enseignant reprend le travail de l'attaque du camp adverse en valorisant les points marqués par les smashs. L'échauffement est un match à 1 contre 1 avec valorisation des smashs, puis le geste technique du smash est remis à l'étude avec l'obligation de viser des zones en smashant. P-ALB replace ce travail du smash dans un contexte de coopération entre partenaires puisqu'il met en place la " tâche expérimentale modifiée » qui consiste à smasher après une réception sur lancer facile. Le but semble être le réinvestissement de l'attaque efficace dans une situation de match.

Le tableau I récapitule les informations données par les enseignants (entretiens) et les données issues de l'observation des séances (interventions verbales). 
Tableau I. Récapitulatif des séances observées à ALB

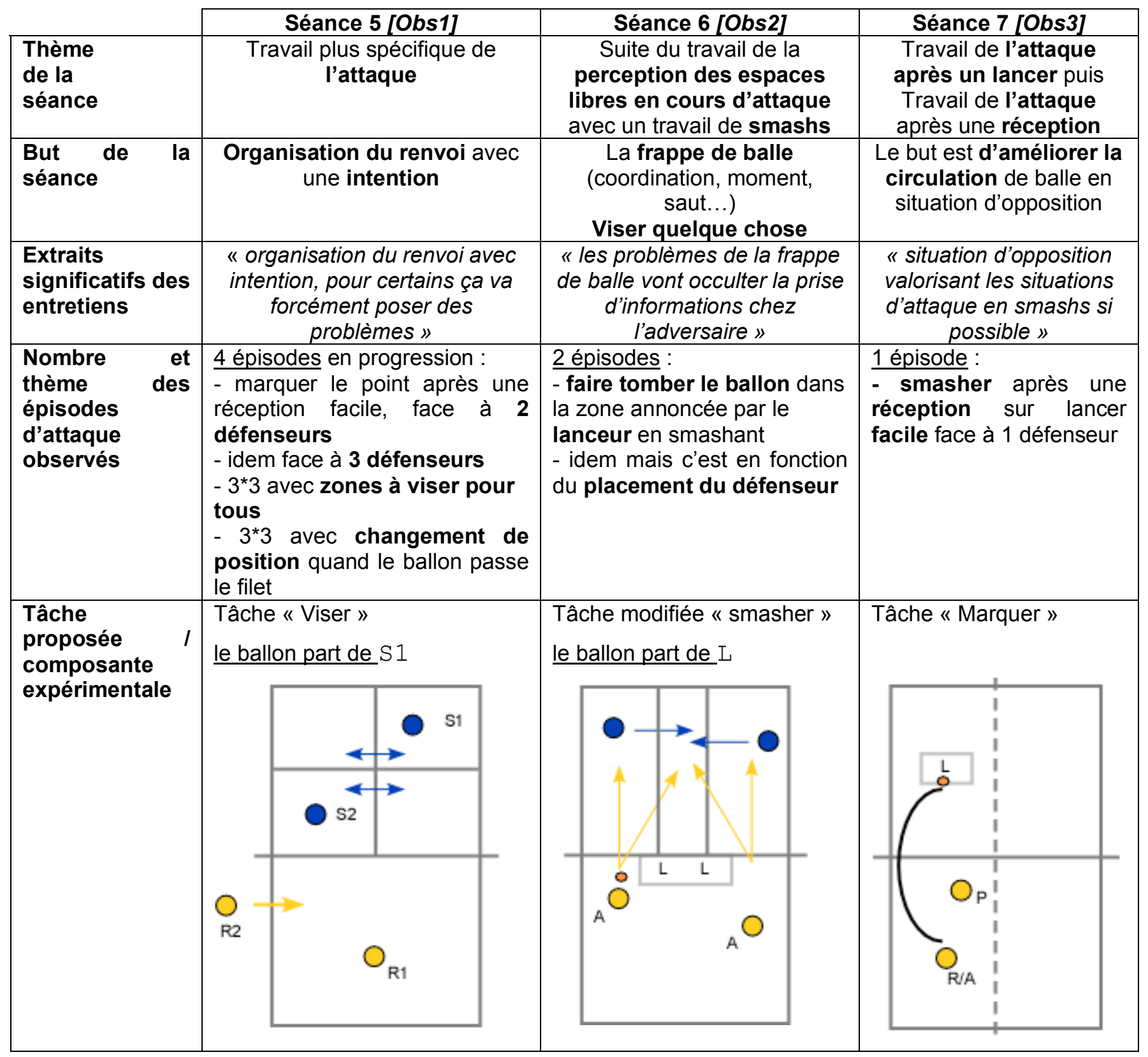

Si l'on se focalise sur les épisodes d'attaque des trois observations, l'enseignant met à l'étude "l'attaque du camp adverse", tout d'abord en l'intégrant dans un jeu de construction en 3 touches de balles, puis en travaillant spécifiquement sur le smash (jouer vite et fort) avant de réinjecter cette " maîtrise " technique du smash dans un jeu de construction en trois touches de balle. La $7^{\text {ème }}$ séance paraît être une sorte de bilan des apprentissages pour P-ALB car au cours de la présentation du thème de cette séance aux élèves, il indique "depuis 2 séances, travail sous forme de précision en jouant dans les espaces libres. Travail pour essayer de prendre l'adversaire de vitesse sous forme de smashs. L'idéal c'est de faire l'amalgame entre les deux: smasher dans les espaces libres : et là, vous êtes vraiment très efficace ». P-ALB se situe dans une conception non 
pas alternative mais cumulative. Nous pensons que beaucoup d'élèves ne tenteront pas de placer le ballon lorsqu'ils ne seront pas en bonne position pour smasher car cette alternative tactique ne fera pas partie de leur registre offensif. Par ailleurs, la centration sur des contenus liés à la coordination course d'élan/impulsion/frappe du ballon, comme ceux liés à l'anticipation-coïncidence renforceront les automatismes de focalisation visuelle sur la trajectoire du ballon, sans les amener à se décentrer pour prendre aussi en compte l'adversaire. La $1^{\text {ère }}$ séance observée qui portait sur l'obligation de "placer la balle », n'était finalement qu'une étape dans la construction du jeu, un préalable sans lien avec le travail du smash. En cours de séance, aucune consigne ne porte sur la nécessité d'observer le jeu adverse. Bien qu'il s'en défende dans les entretiens, il semble que pour le professeur, l'attaque c'est le smash (conception masculine selon la littérature). Nous pensons que c'est cela qui a motivé son abandon de l'attaque placée au profit de deux séances sur le travail technique du geste du smash. L'aide à l'étude de P-ALB valorise le fait de smasher et non sa finalité tactique puisqu'il en vient même, dans les situations d'application (obs.3) à donner comme consigne "je rappelle qu'on marque 3 points lorsqu'on smashe même si on ne réussit pas ». L'analyse met en évidence une disparition de l'alternative au fur et à mesure des séances. II semble que l'enseignant considère que la consigne « renvoyer le ballon dans une des zones où il n'y a pas de joueurs » (attaquer l'espace libre) entraînera de la part des élèves une lecture du comportement de l'adversaire. Or, il n'y a pas cette contrainte dans certaines situations de travail du smash. La lecture de l'adversaire n'est jamais explicitement mise à l'étude. L'enseignant ne fait pas vivre la tâche d'alternative avec sa double composante (attaque placée ou smashée) et l'élève n'a plus de choix à faire. Ce que P-ALB met réellement en place a complètement affaibli les variables didactiques qui permettaient de faire vivre cette tâche, renforçant, sans doute à son insu, les comportements d'attaque dits "plus masculins". Nous pensons à la suite de ces analyses didactiques diachroniques que cette façon de procéder aura sûrement des effets en fonction du genre et du sexe des élèves sur leurs conduites.

\section{2. 2. Trame du cycle et séances observées à AUZ}

Contrairement à ALB, P-AUZ décide de mettre en place les tâches expérimentales dès la $2^{\text {ème }}$ séance de son cycle. Sa conception de l'enseignement vise à faire comprendre aux élèves qu'ils doivent être acteurs de leurs actions et ce quelle que soit l'activité physique. II met en relation les diverses APS enseignées au cours de l'année scolaire et à travers chacune, essaie de provoquer des habitudes de "réflexion sur ce qu'ils peuvent faire " (entretien post). L'idée de faire réfléchir les élèves de façon à ce qu'ils agissent en toute connaissance de cause est dès les premiers contacts, apparue comme un leitmotiv 
(Wirthner \& Schneuwly, 2004) témoignant de sa conception de l'EPS en général et des sports collectifs en particulier (tableau II).

Lors de la 1ère séance, au regard de l'évaluation diagnostique et suite au cycle précédent de badminton où il travaillait sur le fait de "créer l'espace libre " (entretien post-cycle), l'enseignant a insisté sur la notion d'efficacité et sur le fait que les élèves devaient faire des choix en fonction de ce qui arrive. II a également précisé la notion d'intention d'attaque : "qu'ils soient acteurs " (entretien ante). Selon lui, cette séance permettait aux élèves d'identifier leur niveau. Lors de la séance 2 [obs.1] après un échauffement libre, PAUZ aborde la notion d' "efficacité » en volley-ball et propose un exercice de smash dans l'aire de jeu après un lancer (24 min.). L'enjeu est le travail de la coordination course d'élan/impulsion/frappe. A la suite de cet exercice, le professeur met en place la tâche « marquer le point face à deux adversaires ». La séance se termine par un match 4 contre 4 permettant de finaliser les apprentissages. Lors de l'entretien ante, P-AUZ estime que "ça, smasher, ils vont le faire mais est-ce que ça va être dans le terrain?". Dans le même temps il veut " qu'ils [les élèves] se posent la question de qu'est-ce que je vais faire à ce moment-là et pourquoi je fais ça?». Dans cette séance, l'essentiel c'est de comprendre que "l'important n'est finalement pas de smasher mais de marquer des points " (entretien ante). Le thème de la séance 3 [obs.2] était le travail de l'alternative d'attaque. Pendant l'échauffement, les élèves étaient en autonomie. P-AUZ a expliqué la tâche « viser ", mise en place sans modification, et pointé l'intérêt de "réfléchir » avant de frapper. Il a insisté également sur l'importance de «l'intention» (verbatim obs.2)]. Le choix de terminer la séance par des matchs a permis de conserver une bonne dynamique, même si en guise de bilan de fin de séance, l'enseignant dit aux élèves qu'ils n'ont " pas trop fait marcher les méninges ». 
Tableau II. Récapitulatif des séances observées à AUZ

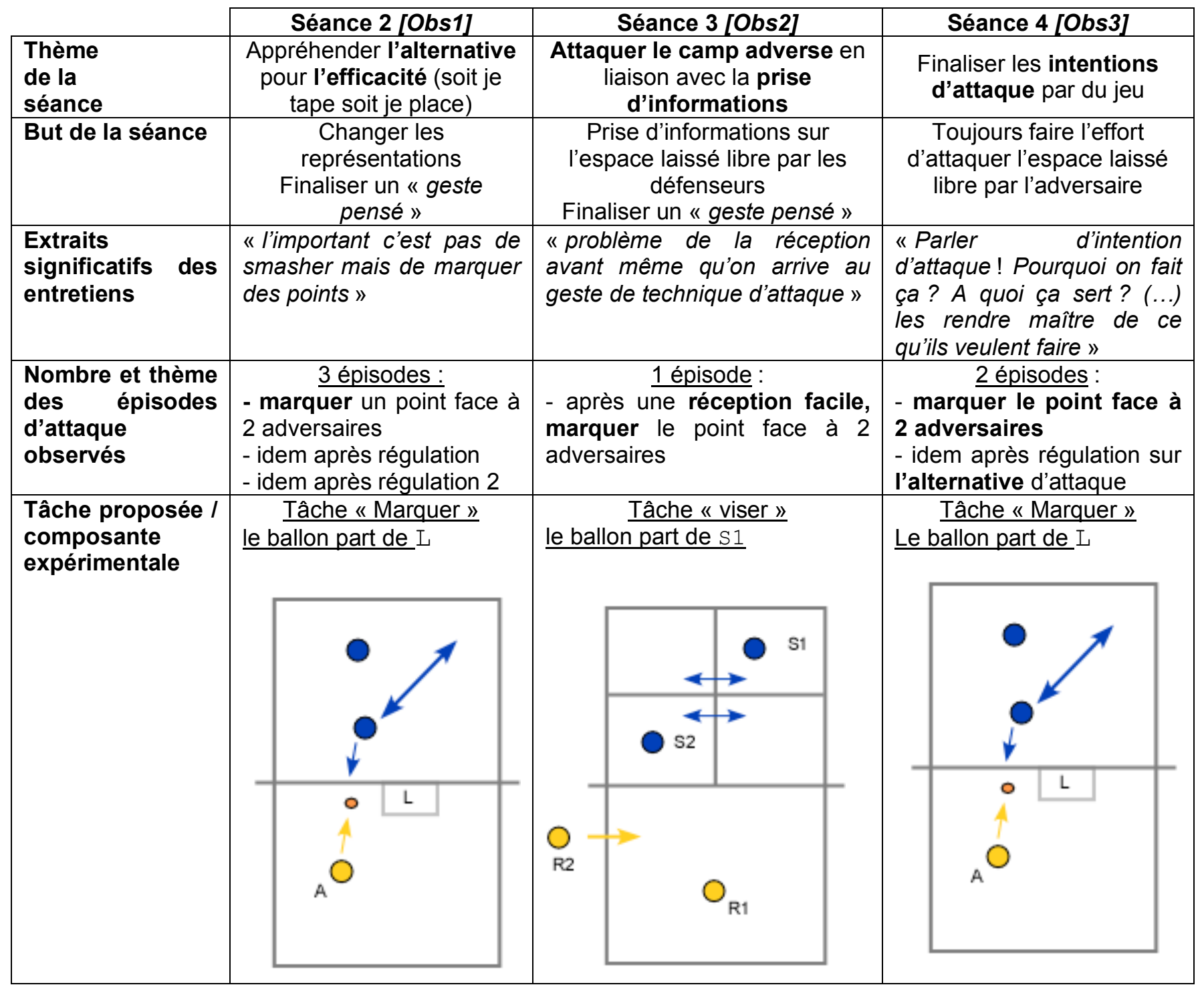

La séance 4 [Obs.3] débute par un échauffement libre puis l'enseignant met en place la tâche expérimentale. Lors de l'entretien ante, P-AUZ précise qu'il a décidé de mettre en place des matchs très tôt dans la séance. Le but est à la fois de vérifier si les élèves réinvestissent en match ce qu'ils ont vu au cours des exercices « c'est le bazar si on finalise pas par du jeu » (entretien ante) [pertinent d'un point de vue didactique], mais surtout pour éviter que les élèves s'ennuient à refaire tout le temps les mêmes exercices « j'ai l'impression qu'ils vont un peu boycotter le système » (entretien ante).

Les épisodes d'attaque mis en place lors des séances observées à AUZ ont pour but de faire prendre conscience aux élèves l'intérêt des intentions tactiques. Ils visent la construction de la notion d'efficacité en attaque, quelle que soit la forme technique d'attaque utilisée, souvent une balle placée. Les premiers épisodes observés correspondent à une situation où l'élève qui attaque doit prendre des informations sur les 
adversaires (tâche " marquer ») tandis que lors de la $2^{\text {ème }}$ séance la prise d'informations sur l'espace libre est valorisée (tâche "viser »). La dernière séance reprend la tâche " marquer face à deux défenseurs » et a pour but de stabiliser les apprentissages qui sont la recherche de l'efficacité, le choix d'attaque (placée dans les espaces libres/frappée) en fonction du ballon reçu et la prise d'informations. Tout au long de ces séances centrées sur l'attaque, P-AUZ réussit à maintenir de façon ingénieuse les 2 composantes de la tâche et donc l'étude d'un savoir tactique : l'alternative d'attaque en fonction de la lecture du positionnement de l'adversaire, de la qualité du ballon reçu et de l'optimisation de ses ressources personnelles.

\section{3. Comparaison des savoirs et des dispositifs mis à l'étude}

Pour rappel, la tâche "viser » consistait à attaquer le ballon en le plaçant dans une des deux zones laissées libres après une réception sur lancer facile. La tâche "marquer » consistait à gagner l'échange face à deux défenseurs sur une balle, envoyé par un lanceur.

Finalement, P-ALB ne met en place que la tâche "viser ». II transforme la tâche « marquer » en un travail de la forme gestuelle du smash doublé d'une obligation de viser un défenseur (et non l'espace libre). Cet enseignant valorise plutôt les savoirs et savoirfaire relatifs à la conclusion de l'attaque, avec primat de la rupture de l'échange en puissance, qui s'apparente aux représentations plutôt masculines de l'activité (Davisse, 1991 ; Tanguy, 1992). P-AUZ pour sa part met plutôt à l'étude les savoirs et les savoirfaire relatifs à la conclusion de l'attaque, avec un plus grand équilibre entre les différentes formes de rupture de l'échange et en mettant l'accent sur l'aspect perceptivo-décisionnel du geste conclusif de l'attaque. D'autre part, ces deux professeurs valorisent, à des degrés divers et bien qu'ils s'en défendent, la composante masculine de l'attaque. Cela est davantage marqué à ALB puisque l'enseignant met en place pendant deux séances un travail gestuel du smash alors que cela se repère de façon indicielle dans les propos de $\mathrm{P}$ AUZ qui péjore parfois cette composante d'attaque lorsqu'il en parle avec ses élèves : "pousser la baballe».

3. 4. Mise en œuvre des tâches concernant l'attaque par les enseignants Du point de vue des épisodes relatifs à la mise en place de l'attaque, à ALB il y a une logique de complication du dispositif didactique tandis qu'à AUZ c'est une logique de sollicitation de la réflexion des élèves. Comme nous l'avons déjà souligné, P-ALB ne met en place que la tâche " viser » et transforme la tâche " marquer " pour travailler la forme gestuelle du smash, en introduisant une variable surprenante eu égard à la logique d'attaque : à la place de viser l'espace libre, l'élève doit viser un défenseur. Une fois les 
situations mises en place, P- AUZ reste vigilant sur l'utilisation des différentes formes de rupture de l'échange et insiste très souvent auprès des élèves sur la prise d'information. II explique souvent qu'un élève qui « fait une bonne prise d'informations avant de frapper la balle, et aura visionné l'endroit où il va la mettre (verbatim obs.2) " aura plus de chances d'être performant. Autre exemple de son insistance sur l'intérêt de prendre des informations pour être efficace : « quelqu'un qui est capable de mettre la balle dans un endroit précis, endroit référencé par rapport à la logique du jeu, j’hésite pas ! Je lui mets 19 ! ॥ (verbatim obs.3).

Ainsi, il s'avère que seul P-AUZ a réellement mis en place les deux tâches, et conservé la valence des tâches expérimentales. P-ALB de son coté met en œuvre un enseignement de l'attaque en volley-ball réduit à la transmission d'une technique alors que l'enseignant du site AUZ développe un enseignement valorisant les aspects tactiques de l'attaque puisqu'il conserve la double composante du protocole expérimental.

\section{Effets de la direction d'étude sur la co-construction des savoirs}

Nous nous situons dans une perspective de didactique comparatiste, souhaitant dépasser les typologies identifiées pour tenter de produire une modélisation des phénomènes différentiels à l'œuvre dans les deux systèmes didactiques étudiés. Dans les recherches comparatistes, les modèles servent à construire et ordonner les faits scientifiques pour suggérer ensuite des hypothèses explicatives qui ne prétendent pas à l'exhaustivité (Vigour, 2005). Selon cette auteure, la modélisation s'appuie sur le constat d'un contraste. Contrairement aux typologies qui expriment un état donné à un «temps $t$ », la modélisation a une fonction dynamique car elle cherche à expliquer la réalité et s'efforce de percevoir la façon dont les processus se déroulent dans le temps. Pour notre part, notre ambition est de contribuer à une meilleure compréhension des processus différentiels à l'œuvre dans l'enseignement de l'attaque en volley-ball. Ainsi, la prise en compte du point de vue didactique de cette étude a consisté à mettre en évidence la dynamique d'évolution des interactions en mettant en relation trois éléments que nous avons tenté de cerner durant nos analyses : les types de milieux didactiques proposés aux élèves, les modalités de direction d'étude des deux enseignants et les formes différentielles de participation des élèves au processus d'enseignement-apprentissage telles que mise en évidence par les chroniques des élèves.

4. 1. Influence des conceptions des enseignants sur leur direction d'étude L'analyse du déroulement des séances observées et son croisement avec les données d'entretiens pointent que P-AUZ réalise un réel travail de l'alternative d'attaque, insistant 
sur l'efficacité dans son aspect tactique. En revanche, P-ALB propose un travail de la forme gestuelle du smash, sans insister sur le repérage des conditions d'efficacité. Pour expliquer les différences dans la mise en œuvre des tâches d'attaque par les enseignants, nous nous sommes intéressées à leur conception respective de l'enseignement de l'attaque en volley-ball. En effet, de précédentes recherches ont montré l'impact des conceptions des sports collectifs sur leur enseignement (Brau-Antony, 2001 ; AmansPassaga, 2005). L'analyse des entretiens croisée avec l'observation des mises en œuvre confirme que l'enseignant P-ALB a une conception plutôt techniciste du volley-ball alors que l'enseignant P-AUZ est plutôt dans une approche dialectique.

Notre interprétation est que P-AUZ met en place les tâches expérimentales telles qu'elles lui ont été soumises, parce qu'elles correspondent à son intention didactique en volley-ball mais également à sa conception de l'apprentissage des sports duels et collectifs. II insiste systématiquement sur l'alternative d'attaque et les solutions existantes pour marquer. II enseigne des savoirs tactiques. En revanche, P-ALB enseigne le travail de la forme gestuelle du smash et choisit de privilégier l'attaque en force (smash). II annonce des solutions possibles pour marquer le point uniquement lors des regroupements en groupeclasse au tableau mais n'aborde pas ce sujet lors des quelques régulations apportées aux élèves. Le temps consacré à l'apprentissage technique du smash mis en place par P-ALB est utile à la maîtrise ultérieure de ce geste, cependant il n'y a pas de mise en relation avec la notion de tactique d'attaque. A contrario, ce temps consacré au travail de la technique gestuelle n'existe pas à AUZ où $P$-AUZ ne met pas en place de tâches consacrées à l'affinement technique du smash.

Au fil des séances observées, la direction d'étude des deux enseignants n'est pas sans effet sur les formes différentielles d'engagement des élèves dans le contrat didactique. Dans le site ALB, les actions des élèves ne sont pas accompagnées de feed-back sur ce qu'ils mettent en œuvre et certains élèves n'arrivent pas à repérer la fonction et le bon moment d'utilisation d'un tel geste. Ils reproduisent une technique gestuelle décontextualisée coupée de l'intention de marquer le point. En revanche P-AUZ intervient systématiquement après chaque action d'élève, que ce soit pour critiquer, réguler, commenter ou valoriser. II ne laisse jamais les élèves en repos concernant la recherche d'efficacité (ce qui est différent de ALB) sans pour cela intervenir sur les modalités concrètes de l'action motrice. Le fait qu'il y ait atténuation de l'alternative d'attaque à ALB au cours des séances met en péril la compréhension par les élèves de son utilité tactique. A l'opposé, P-AUZ ne met jamais l'accent sur le travail de la technique mais insiste beaucoup sur le côté tactique. Au final, les différentes chroniques d'élèves mettent en 
évidence que la plupart des élèves du lycée AUZ semblent avoir construit la notion d'alternative mais, du fait de difficultés techniques, certains n'arrivent pas à la mettre en œuvre, notamment deux filles : Sonia (de genre masculin à I'IRSB et qui appartient à la classe représentationnelle 3, c'est-à-dire des élèves qui veulent progresser pour jouer et attaquer collectivement) et Marion (de genre androgyne à l'IRSB et appartenant à la classe représentationnelle 4 correspondant à des élèves, dont une majorité de filles, qui cherchent à maîtriser le ballon pour le renvoyer). Avec Vigneron (2004), nous pensons qu'un travail de la technique aurait été bénéfique pour les apprentissages de certains élèves et plus particulièrement de ces deux élèves filles. Nous retrouvons là un principe déjà mis en évidence par les recherches sur TGfU selon lequel il convient d'enseigner à la fois les éléments tactiques et techniques du jeu (Kirk, Brooker \& Braiuka, 2000 ; Kirk \& MacPhail, 2002).

Ces différents constats à propos des savoirs enseignés, des dispositifs d'aide à l'étude et de la direction d'étude se recoupent et spécifient chaque système didactique (AUZ et ALB) tout en mettant en évidence des formes différentielles d'acquisitions en relation avec la position de genre des élèves. Nous les présentons de façon succincte dans la section suivante.

4. 2. Modalités de direction d'étude en situation, dynamique des interactions didactiques et effets selon les positions de genre des élèves

Comme indiqué précédemment, nous avons observé la mise en place et l'évolution des deux tâches par chacun des enseignants pendant 3 séances en relation avec les conduites de 16 élèves, filles et garçons (8 sur chaque site), s'inscrivant dans le processus didactique conjoint (Sensevy \& Mercier, 2007) selon des "positions de genre" contrastées (Verscheure \& Amade-Escot, 2007).

Les résultats mettent en évidence qu'il existe des modalités différentes de direction d'études en situation chez ces deux enseignants : P-ALB effectue peu de régulations, qui portent la plupart du temps sur l'aspect organisationnel du milieu. Au contraire, P-AUZ a une grande activité régulatrice, la plupart du temps à propos de l'efficacité des actions tactiques des élèves. Les moments d'enseignement à ALB se font pendant les regroupements au tableau en groupe classe, alors que P-AUZ alterne les moments d'enseignements entre des regroupements en groupe-classe mais aussi lors des petits regroupements, voire de façon individuelle. Lors du regroupement des élèves après quelques minutes d'exercice, P-ALB réduit généralement par ses consignes la tâche « marquer » au travail de la forme gestuelle du smash. II finit par demander aux élèves de smasher systématiquement en visant un défenseur (et non l'espace libre). II valorise ainsi 
plutôt des enjeux de savoir relatifs à la forme gestuelle de l'attaque, en privilégiant la rupture de l'échange en puissance au détriment des composantes tactiques (prise d'information sur les adversaires) inscrites dans le dispositif initial. Cette consigne va avoir pour conséquence une évolution différentielle du contrat didactique selon les élèves filles ou garçons. Reprenant l'exercice, plusieurs garçons tentent de smasher systématiquement et tapent la balle en force avec un engagement indéniable mais ils échouent car ils ne perçoivent pas quelles sont les conditions (hauteur de balle, distance au filet, ajustement postural) qui en permettent la réussite. Certaines filles, notamment les moins habiles se désengagent de la tâche. D'autres se mettent systématiquement à la passe et, de ce fait, tirent finalement peu profit de la situation (Lise-ALB, Charline-ALB). Lors de cette séquence, les interactions didactiques ont ainsi valorisé un mode d'engagement masculin dans l'activité à l'origine des différences d'implication des filles et des garçons Mais au-delà, l'approche techniciste de l'enseignant à propos du volley-ball, le type de tâche qu'il met finalement en place ainsi que sa direction d'étude faiblement régulatrice contribuent à augmenter les écarts à la performance attendue.

Au LEGTA de AUZ, ce sont d'autres éléments qui semblent renforcer les inégalités sexuées entre garçons et filles. En effet, malgré la mise à l'étude de savoirs pertinents (l'attaque dans ses aspects tactiques), P-AUZ développe parfois des comportements paternalistes et méprisants envers certains élèves (filles ou garçons) ce qui peut avoir des conséquences négatives. Cela concerne par exemple Sonia qui a une représentation relativement neutre du volley-ball (résultat de l'enquête préalable) et qui active une position de genre plutôt féminine (Verscheure \& Amade-Escot, 2007). C'est une élève de niveau faible, qui ne trouve pas moyen d'affiner sa technique alors que si elle pouvait le faire elle progresserait. Cette élève est houspillée continuellement par le professeur, ce qui l'amène au fur et à mesure du cycle à perdre confiance en elle. Ainsi, malgré son investissement dans les tâches d'apprentissage, cette élève reste cependant assujettie aux attentes du professeur sans développer une réelle assurance, ce qui explique que ses acquisitions ne trouvent pas de concrétisation en match.

Les conceptions, les mises en œuvre des tâches et les savoirs mis à l'étude ainsi que la direction d'étude du professeur, conjointement avec les élèves, semblent avoir une influence sur les modes d'inscription sexuée dans le contrat didactique différentiel en relation avec la position de genre activée. Sans développer de nouveaux résultats ici, il apparaît que l'action conjointe du professeur et des élèves en situation laisse entrevoir 
trois grands modes d'inscription dans le contrat didactique différentiel selon le genre (Verscheure \& Amade-Escot, 2007).

1) Lors des observations, certains des élèves font preuve d'une grande stabilité relativement aux indicateurs prélevés (sexe, classe représentationnelle, genre/IRSB) et le type de position de genre activée. Par exemple, Rémi (élève AUZ) est de genre nondifférencié à l'IRSB et appartient à la classe représentationnelle 3 (des élèves qui veulent progresser pour jouer et attaquer collectivement). Ses représentations s'actualisent dans la recherche de progrès et de coopération pour attaquer le camp adverse. Cet élève est capable de mettre en œuvre l'alternative d'attaque en prenant en compte le rapport de force avec l'adversaire et ses propres compétences. Cela l'amène dans une position scolaire haute, au plus près des savoirs visés par le professeur. Ainsi, l'entrée en résonance avec le projet didactique du professeur et avec les situations qu'il met en place paraît très importante.

2) Les données recueillies auprès des autres élèves témoignent d'une variation concernant leur profil identitaire. Dans ces cas, l'activation d'une position de genre semble davantage liée aux situations vécues. C'est le cas de Caroline (élève ALB) qui est une bonne illustration de la façon dont des dispositions sociales sexuées peuvent être transformées en dispositions scolaires, dès lors que les milieux didactiques permettent d'actualiser les potentialités. Caroline recherche la maîtrise du ballon pour jouer. Elle décode les enjeux des tâches au-delà des attentes techniques de l'enseignant et, par-là même, s'enseigne à elle-même. Grâce à la prise en compte du rapport de force avec l'adversaire et de ses propres compétences momentanées, elle est capable de mettre en œuvre l'alternative d'attaque. Elle active, in situ, une position de genre selon des modalités équilibrées, ce qui lui permet, en fin de cycle, d'être efficace en attaque en utilisant soit des smashs en puissance soit des attaques placées, en fonction de l'état du rapport de force.

3) Enfin, les positions de genre activées en situation par certains élèves semblent contradictoires relativement aux autres indicateurs prélevés. Par exemple, la dissonance identitaire observée chez Doniphan (ALB) lui permet de tirer profit d'un enseignement centré sur l'attaque smashée. De genre masculin à l'IRSB et appartenant à la classe représentationnelle " des garçons qui cherchent à rompre l'échange à tout prix », il active toutefois de façon intéressante une position de genre équilibrée. En phase avec le contrat didactique qui lui est proposé, il est capable de varier les formes d'attaque, tout en se situant au plus près des intentions didactiques de l'enseignant. Doniphan transforme des dispositions masculines en dispositions scolaires. Un autre exemple est celui de Clélia 
(élève AUZ) qui est de genre féminin mesuré à l'IRSB et développe des représentations masculines du volley-ball. Elle appartient à la classe représentationnelle 2 (des garçons qui recherchent le rapport d'opposition dans un jeu collectif) et cherche la rupture de l'échange en puissance quel que soit le contexte. Nous pensons que son expérience en UNSS a des répercussions négatives sur ses apprentissages. Même si elle active en contexte didactique une position de genre compatible avec ses représentations, elle ne réussit pas à tirer parti des orientations tactiques valorisées par le professeur.

\section{Conclusion}

Au terme de cette comparaison apparaît la complexité à partir de laquelle se structurent les phénomènes observés. L'intentionnalité didactique du professeur, sa conception du savoir à enseigner sont des éléments essentiels dans le processus d'enseignementapprentissage. Dans tous les cas, l'implication des élèves dans les tâches semble liée au type de savoir enseigné et au type de direction d'étude, ce qui corrobore l'idée de la coconstruction de la différence des sexes en classe d'EPS. Dans chaque site, certains élèves trouvent des conditions favorables au développement de leur compétence parce que le contexte interactif et didactique leur permet d'activer des positions de genre (Verscheure \& Amade-Escot, 2007) en adéquation avec les savoirs visés par l'enseignant ou les savoirs qu'ils se fixent eux-mêmes. Dans les deux systèmes didactiques, ces élèves tirent profit de leurs expériences scolaires et ce, malgré les limites de chacun des systèmes (peu d'enseignement tactique, faibles régulations à ALB vs pas de travail de la technique et comportements parfois machistes à AUZ). Dans tous les cas, l'implication des élèves dans les tâches nous semble liée au type de savoir enseigné et au type de direction d'étude (plus qu'aux différences entre un enseignement tactique et un enseignement d'orientation techniciste). Ceci corrobore l'idée de la co-construction de la différence des sexes en classe d'EPS. Les dynamiques mises en évidence soulignent la nécessité d'un enseignement des sports collectifs s'attachant explicitement aux dimensions à la fois techniques et tactiques du jeu si l'on souhaite une plus grande équité dans les acquisitions des filles et des garçons (Verscheure \& Amade-Escot, 2007; Vigneron, 2004). Par ailleurs, même si les mises en œuvre des compétences stratégiques et tactiques sont différentes selon les élèves et leurs " positions de genre " respectives, il est crucial que les formes d'attaques que ces derniers mettent en œuvre, dès lors qu'elles sont efficaces, soient reconnues par les professeurs comme étant de même valeur (Verscheure \& Amade-Escot, 2007). 


\section{Bibliographie}

Amade-Escot, C. (1998). Apport des recherches didactiques à l'analyse de l'enseignement: une étude de cas, le contrat didactique. In C. Amade-Escot, J-P. Barrué, J-C. Bos, F. Dufor, M. Dugrand, A. Terrise (Eds.), Recherches en EPS : Bilan et Perspectives. (pp. 253-2650). Paris : Revue EPS.

Amade-Escot, C. (2001). De l'usage des théories de l'enseignant : questions soulevées par l'étude des contrats didactiques en éducation physique. In A. Mercier, G. Lemoyne, A. Rouchier. Le Génie Didactique. Usages et mésusages des théories de l'enseignement. (pp. 23-41). Bruxelles : De Boeck.

Amade-Escot, C. (2005). Interactions didactiques et difficultés d'apprentissage des filles et des garçons en EPS. In L. Talbot (Dir.). Pratiques d'enseignement et difficultés d'apprentissage (pp. 61-74). Ramonville Ste Agne : Erès.

Amade-Escot, C. (Ed., 2007). Le didactique. Paris : Editions Revue EPS, « Pour l'action ».

Amade-Escot, C. \& Venturini, P. (2009). Ecological and Didactique perspectives on student learning. Paper presented at the AIESEP International Seminar "Situated Learning, Reflective Practice \& Knowledge Construction in Physical Education. Besançon, 27-29 May, France.

Amans-Passaga, C. (2005). L'enseignement des sports collectifs par les professeurs d'EPS dans le cadre de l'association sportive scolaire: étude comparative des conceptions de quelques enseignants. Revue eJRIEPS, 8, 4-23.

Bergé, F. (2004). Mixité en EPS et égalité des genres. Conférence Caen, 31 mars 2004. http://www.contrepied.net/telecharg/berge_mix.pdf.

Brau-Antony, S. (2001). Les conceptions des enseignants d'éducation physique et sportive sur l'enseignement et l'évaluation des jeux sportifs collectifs : résultats d'une enquête, STAPS, 22 (56), 93-108.

Brousseau, G. (1980). L'échec et le contrat. Recherches, 41, 177-182.

Chevallard, Y. (1997). Familière et problématique, la figure du professeur. Recherches en Didactique des Mathématiques, 17/3, 17-54.

Cleuziou, J-P. (2000). L'analyse des menus et des notes au baccalauréat. In B. David (Ed.) EPS : la certification au baccalauréat. (pp. 77-124). Paris : INRP. 
Cogérino, G., Terret, T., \& Rogowski, I. (2006). Mise en place de la mixité en EPS et expertise professionnelle. eJRIEPS, 9, 18-34.

Collectif. (2004). La mixité en question. Contrepied. n¹5. Centre EPS et Société SNEP.

Couchot-Schiex, S. (2007). L'univers de l'enseignant est-il genré ? eJRIEPS, 11, 39-55.

David, B. (1995). Rugby mixte en milieu scolaire. RFP, 110. 51-61.

Davisse, A (1999). «Elles papotent, ils gigotent ». L'indésirable différence des sexes... Ville, Ecole Intégration, 116, 185-198.

Davisse, A., \& Louveau, C. (1991). Sports, école, société : la part des femmes. Paris. Actio.

Duru-Bellat, M. (1994). Filles et garçons à l'école, approches sociologiques et psychosociales. Première partie. Revue Française de Pédagogie, 109, 111-141.

Fize, M. (2003). Les pièges de la mixité scolaire. Paris : Presses de la renaissance.

Fontayne, P., Sarrazin, P., \& Famose, J.-P. (2000). The Bem Sex-Role Inventory: Validation of a short-version for French teenagers. European Review of Applied Psychology, 50 (4), 405-417.

Fontayne, P., Sarrazin, P. \& Famose, J-P. (2001). Les pratiques sportives des adolescents : une différenciation selon le genre. STAPS, 55, 23-27.

Goirand, P. (1998). EPS au collège et gymnastique. Paris : INRP.

Huberman, M., \& Miles, M.B. (1991). Analyse des données qualitatives : recueil de nouvelles méthodes. Bruxelles : De Boëck.

Johsua, S., \&, Lahire, B. (1999). Pour une didactique sociologique (dossier Sociologie des savoirs). Education et Société. 4. 29-56.

Griffin, P.S. (1984). Girls' participation patterns in a middle school team sports unit. Journal of Teaching in Physical Education, 4(1), 30-38.

Griffin, P.S. (1985). Boys' participation styles in a middle school physical education team sports unit. Journal of Teaching in Physical Education, 4(2), 100-110.

Harris, J. (1997). 'Physical education: A Picture of Health? The Implement of HealthRelated exercise in the National Curriculum in Secondary Schools in England'. Unpublished Doctoral Thesis, Loughborough University. 
Harris, J., \& Penney, D. (2000). Gender issues in health-related exercise. European Physical Education Review. 6/3. 249-273.

Hastie, P., \& Siedentop, D. (2006). The classroom ecology paradigm, In D. Kirk, M. O'Sullivan, and D. Macdonald (Eds.). Handbook of Research in Physical Education (pp. 214-225). London, Thousand Oaks, New Delhi: SAGE Publications Ltd.

Kirk, D. (2002). Student learning and the social construction of gender in sport and physical education. in J. Silverman, C.D. Ennis. Student Learning in Physical Education (pp. 67-82). (2nd edition) Human Kinetics.

Kirk, D., Brooker, R., \& Braiuka, S. (2000) Teaching Games for Understanding: A Situated Perspective on Student Learning. AERA. Annual Meeting. New Orleans, USA. April.

Kirk, D., \& MacPhail, A. (2002). Teaching games for understanding and situated learning: rethinking the Bunker-Thorpe model. Journal of Teaching in Physical Education, 21, 177-192.

Lessard-Hebert, M., Goyette, G., \&, Boutin, G. (1996). La recherche qualitative. Fondements et pratiques. Montréal : Editions Nouvelles (2èm ed.).

Leutenegger, F. (2003). Etude des interactions didactiques en classe de mathématiques : un prototype méthodologique. In A. Danis, M.L. Schubauer-Leoni \& A. Weil-Barais (Ed.), Interaction, Acquisition de connaissances et Développement. Bulletin de Psychologie 56 (4) 466, 559-571.

Luke, M.D., \& sinclair, G.D. (1991). Gender differences in adolescents' attitudes toward physical education. Journal of Teaching in Physical education,11, 31-46

Macdonald, D. (1990). The Relationship Between the Sex Composition of Physical Education Classes and Teacher/Pupil Verbal Interaction. Journal of Teaching in Physical Education, 9. 152-163.

Marsenach, J. (1995). EPS au collège et volley-ball. Paris : INRP.

Marsenach, J. (2004). Pour des contenus ambitieux. Revue Contre Pied, 15. 42.

Marsenach, J., \&, Mérand, R. (1987). L'évaluation formative en EPS dans les collèges, INRP, Paris.

Mercier A., \& Salin M-H., (1988). L'analyse a priori, outil pour l'observation. Actes "Didactique et formation des maîtres à l'Ecole Elémentaire". Bordeaux : IREM. 
Ministère de l'Education Nationale. (2000). Programmes des enseignements de la classe de seconde générale et technologique : EPS. Hors-Série nº 6 du 31 août 2000.

Mosconi, N., \&, Loudet-Verdier, J. (1997). Inégalités de traitement entre les filles et les garçons. In C., Blanchard-Laville, Variations sur une leçon de mathématiques. (pp. 127-150). Paris. L'Harmattan.

Mosconi, N. (2003). Rapport au savoir et division socio-sexuée des savoirs à l'école. La Lettre du GRAPE, 51, 31-38.

Schubauer-Léoni, M.L. (1988). Maître-élève-savoir : analyse psychosociale du jeu et des enjeux de la relation didactique. Thèse de Doctorat, non publiée. Faculté de psychologie et des sciences de l'éducation. Genève.

Schubauer-Leoni, M.L. (1996). Etude du contrat didactique pour des élèves en difficulté en mathématiques. Problématique didactique et/ou psychosociale. In C. Raisky, et $\mathrm{M}$. Caillot (Eds.) Au-delà des didactiques le didactique : débats autour de concepts fédérateurs (pp. 159-189). Bruxelles: De Boëck, Perspectives en éducation.

Schubauer-Leoni, M.L. (2002). Didactique comparée et représentations sociales. In AFIRSE (Ed.) L'année de la recherche en sciences de l'éducation (pp. 127-149). Paris : Afirse, Matrice.

Scraton, S. (1986). Images of Femininity and the teaching of Girls Physical Education. In J. Evans, (ed.) Physical Education; Sport and Schooling: Studies in the Sociology of Physical Education. (pp. 71-94). Lewes: Falmer Press.

Sensevy, G., \& Mercier, A. (2007). Agir ensemble : Eléments de théorisation de l'action conjointe du professeur et des élèves. Rennes : PUR.

Tanguy, G. (1992). Le volley: un exemple de mise en œuvre didactique. Echanges et controverses. 4. 7-20.

Verscheure, I., \& Amade-Escot, C. (2004). Dynamiques différentielles des interactions didactiques selon le genre en EPS. Le cas de l'attaque en volley-ball en seconde. STAPS, 66, 79-97.

Verscheure, I., \& Amade-Escot, C. (2007). The gendered construction of physical education content as the result of the differentiated didactic contract. Physical Education and Sport Pedagogy, 12(3), 245-272. 
Verscheure, I., \& Amade-Escot, C., Chiocca, C-M. (2006). Représentations du volley-ball scolaire et genre des élèves: Pertinence de l'IRSB ? Revue Française de Pédagogie, 154, 125-141.

Vigneron, C. (2004). La construction des inégalités de réussite en EPS au baccalauréat entre filles et garçons. Thèse de doctorat en Sciences de l'Education. Non publiée. Université de Bourgogne. France.

Vigour, C. (2005). La comparaison dans les sciences sociales. Pratiques et méthodes. Paris : La découverte.

Wirthner, M., \& Schneuwly, B., (2004). Variabilité et contrainte dans la construction des significations d'un objet d'enseignement. L'effet d'un outil pour enseigner le résumé d'un texte informatif. in C., Moro, R. Rickerman (Eds). Situation éducative et significations. (pp. 107-133). Bruxelles : De Boeck et Larcier.

Whitson, D. (1994). The embodiment of gender: discipline, domination and empowerment, In S. Birrell, S. Cole (Eds.), Women, Sport and Culture (pp. 353-371).Champaign; II: Human Kinetics.

Wright, J. (1997). The Construction of Gendered Contexts in Single Sex and Coeducational Physical Education Lessons. Sport, Education and Society. 2/1. 55-72. 